\title{
DECIDABLE EXPANSIONS OF LABELLED LINEAR ORDERINGS
}

\author{
ALEXIS BÈS $^{a}$ AND ALEXANDER RABINOVICH ${ }^{b}$ \\ ${ }^{a}$ University of Paris-Est Créteil, LACL \\ e-mail address: bes@u-pec.fr \\ ${ }^{b}$ Tel-Aviv University, The Blavatnik School of Computer Science \\ e-mail address: rabinoa@post.tau.ac.il
}

\begin{abstract}
Consider a linear ordering equipped with a finite sequence of monadic predicates. If the ordering contains an interval of order type $\omega$ or $-\omega$, and the monadic secondorder theory of the combined structure is decidable, there exists a non-trivial expansion by a further monadic predicate that is still decidable.
\end{abstract}

\section{INTRODUCTION}

In this paper we address definability and decidability issues for monadic second order (shortly: MSO) theories of labelled linear orderings. Elgot and Rabin ask in [10] whether there exist maximal decidable structures, i.e., structures $M$ with a decidable first-order (shortly: FO) theory and such that the FO theory of any expansion of $M$ by a non-definable predicate is undecidable. This question is still open. Let us mention some partial results:

- Soprunov proved in [30] that every structure in which a regular ordering is interpretable is not maximal. A partial ordering $(B,<)$ is said to be regular if for every $a \in B$ there exist distinct elements $b_{1}, b_{2} \in B$ such that $b_{1}<a, b_{2}<a$, and no element $c \in B$ satisfies both $c<b_{1}$ and $c<b_{2}$. As a corollary he also proved that there is no maximal decidable countable structure if we replace FO by weak MSO logic.

- In [2], Bès and Cégielski consider a weakening of the Elgot-Rabin question, namely the question of whether all structures $M$ whose FO theory is decidable can be expanded by some constant in such a way that the resulting structure still has a decidable theory. They answer this question negatively by proving that there exists a structure $M$ with a decidable MSO theory and such that any expansion of $M$ by a constant has an undecidable FO theory.

2000 ACM Subject Classification: F.4.1 F.4.3.

Key words and phrases: Monadic second-order logic, decidability, definability, linear orderings.

A preliminary version of this paper appeared in [3].

${ }^{a, b}$ This research was facilitated by the ESF project AutoMathA. The second author was partially supported by ESF project Games and EPSRC grant.

LOGICAL METHODS IN COMPUTER SCIENCE
DOI:10.2168/LMCS-7 (2:5) 2011

(C) A. Bès and A. Rabinovich (c) Creative Commons 
- The paper [1] gives a sufficient condition in terms of the Gaifman graph of $M$ which ensures that $M$ is not maximal. The condition is the following: for every natural number $r$ and every finite set $X$ of elements of the base set $|M|$ of $M$ there exists an element $x \in|M|$ such that the Gaifman distance between $x$ and every element of $X$ is greater than $r$.

We investigate the Elgot-Rabin problem for the class of labelled linear orderings, i.e., infinite structures $M=\left(A ;<, P_{1}, \ldots, P_{n}\right)$ where $<$ is a linear ordering over $A$ and the $P_{i}$ 's denote unary predicates. This class is interesting with respect to the above results, since on one hand no regular ordering seems to be FO interpretable in such structures, and on the other hand their associated Gaifman distance is trivial, thus they do not satisfy the criterion given in 1 .

In this paper we focus on MSO logic rather than FO. The main result of the paper is that for every labelled linear ordering $M$ such that $(A,<)$ contains an interval of order type $\omega$ or $-\omega$ and the MSO theory of $M$ is decidable, there exists an expansion $M^{\prime}$ of $M$ by a monadic predicate which is not MSO-definable in $M$, and such that the MSO theory of $M^{\prime}$ is still decidable. Hence, $M$ is not maximal. The result holds in particular when $(A,<)$ is order-isomorphic to the order of the naturals $\omega=(\mathbb{N},<)$, or to the order $\zeta=(\mathbb{Z},<)$ of the integers, or to any infinite ordinal, or more generally any infinite scattered ordering (recall that an ordering is scattered if it does not contain any dense sub-ordering).

The structure of the proof is the following: we first show that the result holds for $\omega$ and $\zeta$. For the general case, starting from $M$, we use some definable equivalence relation on $A$ to cut $A$ into intervals whose order type is either finite, or of the form $-\omega, \omega$, or $\zeta$. We then define the new predicate on each interval (using the constructions given for $\omega$ and $\zeta$ ), from which we get the definition of $M^{\prime}$. The reduction from $M S O\left(M^{\prime}\right)$ to $M S O(M)$ uses Shelah's composition theorem, which allows us to reduce the MSO theory of an ordered sum of structures to the MSO theories of the summands.

The main reason to consider MSO logic rather than FO is that it actually simplifies the task. Nevertheless we discuss some partial results and perspectives for FO logic in the conclusion of the paper.

Let us recall some important decidability results for MSO theories of linear orderings (the case of labelled linear orderings will be discussed later for $\omega$ and $\zeta$ ). In his seminal paper [5], Büchi proved that languages of $\omega$-words recognizable by automata coincide with languages definable in the MSO theory of $\omega$, from which he deduced decidability of the theory. The result (and the automata method) was then extended to the MSO theory of any countable ordinal [6], to $\omega_{1}$, and to any ordinal less than $\omega_{2}$ [7]. Gurevich, Magidor and Shelah prove [15] that decidability of MSO theory of $\omega_{2}$ is independent of ZFC. Let us mention results for linear orderings beyond ordinals. Using automata, Rabin 21 proved decidability of the MSO theory of the binary tree, from which he deduces decidability of the MSO theory of $\mathbb{Q}$, which in turn implies decidability of the MSO theory of the class of countable linear orderings. Shelah [28] improved model-theoretical techniques that allowed him to reprove almost all known decidability results about MSO theories, as well as new decidability results for the case of linear orderings, and in particular dense orderings. He proved in particular that the MSO theory of $\mathbb{R}$ is undecidable. The frontier between decidable and undecidable cases was specified in later papers by Gurevich and Shelah [13, 16, 17]; we refer the reader to the survey [14]. 
Our result is also clearly related to the problem of building larger and larger classes of structures with a decidable MSO theory. For an overview of recent results in this area see [4, 34].

\section{Preliminaries}

1.1. Labelled Linear Orderings. We first recall useful definitions and results about linear orderings. A good reference on the subject is Rosenstein's book [25].

A linear ordering $J$ is a total ordering. We denote by $\omega$ (respectively $\zeta$ ) the order type of $\mathbb{N}$ (respectively $\mathbb{Z}$ ). Given a linear ordering $J$, we denote by $-J$ the backwards linear ordering obtained by reversing the ordering relation.

Given a linear ordering $J$ and $j \in J$, we denote by $[j]$ the interval $[j, j]$. An ordering is dense if it contains no pair of consecutive elements. An ordering is scattered if it contains no dense sub-ordering.

In this paper we consider labelled linear orderings, i.e., linear orderings $(A,<)$ equipped with a function $f: A \rightarrow \Sigma$ where $\Sigma$ is a finite nonempty set.

1.2. Logic. Let us briefly recall useful elements of monadic second-order logic, and settle some notations. For more details about MSO logic see e.g. [14, 33. Monadic secondorder logic is an extension of first-order logic that allows to quantify over elements as well as subsets of the domain of the structure. Given a signature $L$, one can define the set of (MSO) formulas over $L$ as well-formed formulas that can use first-order variable symbols $x, y, \ldots$ interpreted as elements of the domain of the structure, monadic secondorder variable symbols $X, Y, \ldots$ interpreted as subsets of the domain, symbols from $L$, and a new binary predicate $x \in X$ interpreted as " $x$ belongs to $X$ ". A sentence is a formula without free variable. As usual, we often confuse logical symbols with their interpretation. Given a signature $L$ and an $L$-structure $M$ with domain $D$, we say that a relation $R \subseteq$ $D^{m} \times\left(2^{D}\right)^{n}$ is (MSO) definable in $M$ if and only if there exists a formula over $L$, say $\varphi\left(x_{1}, \ldots, x_{m}, X_{1}, \ldots, X_{n}\right)$, which is true in $M$ if and only if $\left(x_{1}, \ldots, x_{m}, X_{1}, \ldots, X_{n}\right)$ is interpreted by an $(m+n)$-tuple of $R$. Given a structure $M$ we denote by $M S O(M)$ (respectively $F O(M)$ ) the monadic second-order (respectively first-order) theory of $M$. We say that $M$ is maximal if $M S O(M)$ is decidable and $M S O\left(M^{\prime}\right)$ is undecidable for every expansion $M^{\prime}$ of $M$ by a predicate which is not definable in $M$.

We can identify labelled linear orderings with structures of the form $M=(A,<$ $\left., P_{1}, \ldots, P_{n}\right)$ where $<$ is a binary relation interpreted as a linear ordering over $A$, and the $P_{i}$ 's denote unary predicates. We use the notation $\bar{P}$ as a shortcut for the $n$-tuple $\left(P_{1}, \ldots, P_{n}\right)$. The structure $M$ can be seen as a word indexed by $A$ and over the alphabet $\Sigma_{n}=\{0,1\}^{n}$; this word will be denoted by $w(M)$. For every interval $I$ of $A$ we denote by $M_{I}$ the sub-structure of $M$ with domain $I$.

Let $\Sigma$ and $\Sigma^{\prime}$ be relational signatures, $M$ a $\Sigma$-structure with domain $A$ and $M^{\prime}$ a $\Sigma^{\prime}$ structure with domain $A^{\prime}$. We say that $M$ is (MSO) interpretable in $M^{\prime}$ if there exist a subset $D$ of $A^{\prime}$ and a surjective map $\mathcal{I}: D \rightarrow A$ such that:

- $D$ is MSO definable in $M^{\prime}$;

- The equivalence relation $E Q_{\mathcal{I}}=\left\{(x, y) \in A^{\prime}: \mathcal{I}(x)=\mathcal{I}(y)\right\}$ is MSO definable in $M^{\prime}$; 
- For every $m$-ary symbol $R$ of $\Sigma$, there exists a MSO $\Sigma^{\prime}$-formula $\varphi_{R}$ such that

$$
M \models R\left(\mathcal{I}\left(a_{1}\right), \ldots, \mathcal{I}\left(a_{m}\right)\right) \Leftrightarrow M^{\prime} \models \varphi_{R}\left(a_{1}, \ldots, a_{m}\right)
$$

for all $a_{1}, \ldots, a_{m} \in D$.

The following property of interpretations is well-known.

Lemma 1.1. If $M$ is interpretable in $M^{\prime}$ then $M S O(M)$ is recursive in $M S O\left(M^{\prime}\right)$.

1.3. Elements of the Composition Method. In this paper we rely heavily on composition methods, which allow us to compute the theory of a sum of structures from the ones of its summands. For an overview of the subject see [4, 32, 18]. In this section we recall useful definitions and results.

The quantifier depth of a formula $\varphi$ is denoted by $q d(\varphi)$. Let $n \in \mathbb{N}, \Delta$ any finite signature that contains only relational symbols, and $M_{1}, M_{2}$ be $\Delta$-structures. We say that $M_{1}$ and $M_{2}$ are $n$-equivalent, denoted $M_{1} \equiv^{n} M_{2}$, if for every sentence $\varphi$ of quantifier depth at most $n, M_{1} \models \varphi$ iff $M_{2} \models \varphi$.

Clearly, $\equiv^{n}$ is an equivalence relation. For any $n \in \mathbb{N}$ and $\Delta$, the set of sentences of quantifier depth $\leq n$ is infinite. However, it contains only finitely many semantically distinct sentences, so there are only finitely many $\equiv^{n}$-classes of $\Delta$-structures. In fact, we can compute representatives for these classes.

Lemma 1.2 (Hintikka Lemma). For each $n \in \mathbb{N}$ and a finite signature $\Delta$ that contains only relational symbols, we can compute a finite set $H_{n}(\Delta)$ of $\Delta$-sentences of quantifier depth at most $n$ such that:

- If $\tau_{1}, \tau_{2} \in H_{n}(\Delta)$ and $\tau_{1} \neq \tau_{2}$, then $\tau_{1} \wedge \tau_{2}$ is unsatisfiable.

- If $\tau \in H_{n}(\Delta)$ and $q d(\varphi) \leq n$, then either $\tau \rightarrow \varphi$ or $\tau \rightarrow \neg \varphi$. Furthermore, there is an algorithm that, given such $\tau$ and $\varphi$, decides which of these two possibilities holds.

- For every $\Delta$-structure $M$ there is a unique $\tau \in H_{n}(\Delta)$ such that $M \models \tau$.

Elements of $H_{n}(\Delta)$ are called $(n, \Delta)$-Hintikka sentences.

Given a $\Delta$-structure $M$ we denote by $T^{n}(M)$ the unique element of $H_{n}(\Delta)$ satisfied in $M$ and call it the $n$-type of $M$. Thus, $T^{n}(M)$ determines (effectively) which sentences of quantifier-depth $\leq n$ are satisfied in $M$.

As a simple consequence, note that the MSO theory of a structure $M$ is decidable if and only if the function $k \mapsto T^{k}(M)$ is recursive.

The sum of structures corresponds to concatenation; let us give a general definition.

Definition 1.3 (sum of chains). Consider an index structure $\operatorname{Ind}=\left(I,<^{I}\right)$ where $<^{I}$ is a linear ordering. Consider a signature $\Delta=\left\{<, P_{1}, \ldots, P_{l}\right\}$, where $P_{i}$ are unary predicate names, and a family $\left(M_{i}\right)_{i \in I}$ of $\Delta$-structures $M_{i}=\left(A_{i} ;<^{i}, P_{1}{ }^{i}, \ldots, P_{l}^{i}\right)$ with disjoint domains and such that the interpretation $<^{i}$ of $<$ in each $M_{i}$ is a linear ordering. We define the ordered sum of the family $\left(M_{i}\right)_{i \in I}$ as the $\Delta$-structure $M=\left(A ;<^{M}, P_{1}{ }^{M}, \ldots, P_{l}^{M}\right)$ where

- $A$ equals the union of the $A_{i}$ 's

- $x<{ }^{M} y$ holds if and only if $\left(x \in A_{i}\right.$ and $y \in A_{j}$ for some $\left.i<{ }^{I} j\right)$, or $\left(x, y \in A_{i}\right.$ and $\left.x<{ }^{i} y\right)$

- for every $x \in A$ and every $k \in\{1, \ldots, l\}, P_{k}^{M}(x)$ holds if and only if $M_{j} \models P_{k}^{j}(x)$ where $j$ is such that $x \in A_{j}$. 
If the domains of the $M_{i}$ are not disjoint, replace them with isomorphic chains that have disjoint domains, and proceed as before.

We shall use the notation $M=\sum_{i \in I} M_{i}$ for the ordered sum of the family $\left(M_{i}\right)_{i \in I}$.

If $I=\{1,2\}$ has two elements, we denote $\sum_{i \in I} M_{i}$ by $M_{1}+M_{2}$.

We need the following composition theorem on ordered sums (see e.g. [32]):

\section{Theorem 1.4.}

(a) The $k$-types of labelled linear orderings $M_{0}, M_{1}$ determine the $k$-type of the ordered sum $M_{0}+M_{1}$, which moreover can be computed from the $k$-types of $M_{0}$ and $M_{1}$.

(b) If the labelled linear orderings $M_{0}, M_{1}, \ldots$ all have the same $k$-type, then this $k$-type determines the $k$-type of $\Sigma_{i \in \mathbb{N}} M_{i}$, which moreover can be computed from the $k$-type of $M_{0}$.

Part (a) of the theorem justifies the notation $s+t$ for the $k$-type of a linear ordering which is the sum of two linear orderings of $k$-types $s$ and $t$, respectively. Similarly, we write $t \times \omega$ for the $k$-type of a sum $\Sigma_{i \in \mathbb{N}} M_{i}$ where all $M_{i}$ have $k$-type $t$.

For every linear ordering $(A,<)$ and every $x \in A$ let $I_{<x}=\{y \in A: y<x\}$ and $I_{\geq x}=\{y \in A: y \geq x\}$. The following is a well-known consequence of Theorem 1.4(a) (see e.g [12, Theorem A.1]).

Corollary 1.5. Let $M=(A,<, \bar{P}), k \geq 1$, and $a, b \in A$ be such that $I_{<a}$ and $I_{<b}$ are nonempty sets. Assume that $T^{k}\left(M_{I_{<a}}\right)=T^{k}\left(M_{I_{<b}}\right)$ and $T^{k}\left(M_{I_{\geq a}}\right)=T^{k}\left(M_{I_{\geq b}}\right)$. Then for every formula $\varphi(x)$ such that $q d(\varphi)<k$, we have

$$
M \models \varphi(a) \text { if and only if } M \models \varphi(b) .
$$

We shall use the following result.

Proposition 1.6. Let $M=(A,<, \bar{P}), I$ be an interval of $A$, and $Q \subseteq A$. If $Q$ is definable in $M$ then $Q \cap I$ is definable in $M_{I}$.

Proof. Assume that the formula $\varphi(x)$ defines $Q$ in $M$. Note that $Q$ is the unique predicate which satisfies the formula $\psi(X) \equiv \forall x(x \in X \leftrightarrow \varphi(x))$. Let $m=q d(\psi)$. For every $R \subseteq A$, let us denote by $M^{R}$ the expansion of $M$ with a new monadic predicate $\mathbf{X}$ interpreted by $R$. By our hypothesis, we have $T^{m}\left(M^{R}\right)=T^{m}\left(M^{Q}\right)$ if and only if $Q=R$.

Let $J_{1}$ (respectively $J_{2}$ ) be the set of elements of $A$ less than (respectively greater than) every element of $I$. We have $M^{R}=M_{J_{1}}^{R}+M_{I}^{R}+M_{J_{2}}^{R}$. We claim that $T^{m}\left(M_{I}^{R}\right)=T^{m}\left(M_{I}^{Q}\right)$ if and only if $R \cap I=Q \cap I$. Indeed assume for a contradiction that there exists $R$ such that $T^{m}\left(M_{I}^{R}\right)=T^{m}\left(M_{I}^{Q}\right)$ and $R \cap I \neq Q \cap I$. Consider $Q^{\prime} \subseteq A$ such that $Q^{\prime} \cap J_{1}=Q \cap J_{1}$, $Q^{\prime} \cap J_{2}=Q \cap J_{2}$ and $Q^{\prime} \cap I=R \cap I$. On the one hand we have $Q^{\prime} \neq Q$, and on the other hand by Theorem 1.4(a) we have

$T^{m}\left(M^{Q^{\prime}}\right)=T^{m}\left(M_{J_{1}}^{Q}\right)+T^{m}\left(M_{I}^{R}\right)+T^{m}\left(M_{J_{2}}^{Q}\right)=T^{m}\left(M_{J_{1}}^{Q}\right)+T^{m}\left(M_{I}^{Q}\right)+T^{m}\left(M_{J_{2}}^{Q}\right)=T^{m}\left(M^{Q}\right)$ which contradicts the fact that $Q$ is the unique predicate which satisfies $\psi(X)$ in $M$.

The type $T^{m}\left(M_{I}^{Q}\right)$ is a sentence $\theta$ over the signature $\{<, \bar{P}\} \cup\{\mathbf{X}\}$. We can see it as a formula $\theta(X)$ with one free monadic variable $X$ over the signature $\{<, \bar{P}\}$. We have $M_{I} \models \theta(X)$ if and only if $X=Q \cap I$. Thus the formula $\gamma(x)$ defined as $\exists X(\theta(X) \wedge x \in X)$ defines $Q \cap I$ in $M_{I}$. 
1.4. Decomposition of a labelled linear ordering. Let $M=(A,<, \bar{P})$ be a labelled linear ordering and let $\sim$ be an equivalence relation on $A$. If the $\sim$-equivalence classes are intervals of $A$ we say that $\sim$ is a convex equivalence relation. In this case the set of $\sim$-equivalence classes can be naturally ordered by $i_{1} \leq i_{2}$ iff $\exists x_{1} \in i_{1} \exists x_{2} \in i_{2}\left(x_{1} \leq x_{2}\right)$. We denote by $M / \sim$ the linear order of $\sim$-equivalence classes. The mapping that assigns to every $x \in A$ its $\sim$-equivalence class is said to be canonical.

Let $\sim$ be a convex equivalence relation on $A$. Then $M=\sum_{i \in M / \sim} M_{i}$, where $M_{i}$ is the substructure of $M$ with domain the equivalence class $i$.

Lemma 1.7. If $\sim$ is a convex equivalence relation which is definable in $M$, then

(1) $M / \sim$ is interpretable in $M$.

(2) Let $\varphi_{1}, \ldots \varphi_{k}$ be sentences in the signature of $M$. Let

$$
N=\left(M / \sim,<, Q_{\varphi_{1}}, \ldots, Q_{\varphi_{k}}\right)
$$

where $Q_{\varphi_{l}}=\left\{i \in M / \sim \mid M_{i} \models \varphi_{l}\right\}$ for every l. Then $N$ is interpretable in $M$.

\section{THE CASE OF $\mathbb{N}$}

In this section we prove that there is no maximal structure of the form $(\mathbb{N},<, \bar{P})$ with respect to MSO logic. The proof is based upon results from [22] . Let us first briefly review results related to the decidability of the MSO theory of expansions of $(\mathbb{N},<)$. Büchi $[5$ ] proved decidability of $M S O(\mathbb{N},<)$ using automata. On the other hand it is known that $M S O(\mathbb{N},+)$, and even $M S O(\mathbb{N},<, x \mapsto 2 x)$, are undecidable [24]. Elgot and Rabin study in [10] the MSO theory of structures of the form $(\mathbb{N},<, P)$, where $P$ is some unary predicate. They give a sufficient condition on $P$ which ensures decidability of the MSO theory of $(\mathbb{N},<, P)$. In particular the condition holds when $P$ denotes the set of factorials, or the set of powers of any fixed integer. The frontier between decidability and undecidability of related theories was explored in numerous later papers $[8,11,27,26,23,22,29$, , 31. Let us also mention that [27] proves the existence of unary predicates $P$ and $Q$ such that both $M S O(\mathbb{N},<, P)$ and $M S O(\mathbb{N},<, Q)$ are decidable while $M S O(\mathbb{N},<, P, Q)$ is undecidable.

Most decidability proofs for $M S O(\mathbb{N},<, \bar{P})$ are related somehow to the possibility of cutting $\mathbb{N}$ into segments whose $k$-type is ultimately constant, from which one can compute the $k$-type of the whole structure (using Theorem 1.4). This connection was specified in [22] (see also [23]) using the notion of homogeneous sets.

Definition 2.1 ( $k$-homogeneous set). Let $k \geq 0$. A set $H=\left\{h_{0}<h_{1}<\ldots\right\} \subseteq \mathbb{N}$ is called $k$-homogeneous for $M=(\mathbb{N},<, \bar{P})$, if all sub-structures $M_{\left[h_{i}, h_{j}[\right.}$ for $i<j$ (and hence all sub-structures $M_{\left[h_{i}, h_{i+1}[\right.}$ for $\left.i \geq 0\right)$ have the same $k$-type.

This notion can be refined as follows.

Definition 2.2 (uniformly homogeneous set). A set $H=\left\{h_{0}<h_{1}<\ldots\right\} \subseteq \mathbb{N}$ is called uniformly homogeneous for $M=(\mathbb{N},<, \bar{P})$ if for each $k$ the set $H_{k}=\left\{h_{k}<h_{k+1}<\ldots\right\}$ is $k$-homogeneous.

The following result [22] settles a tight connection between $M S O(\mathbb{N},<, \bar{P})$ and uniformly homogeneous sets. 
Theorem 2.3. ([22]) For every $M=(\mathbb{N},<, \bar{P})$, the $M S O$ theory of $M$ is decidable if and only if (the sets $\bar{P}$ are recursive and there exists a recursive uniformly homogeneous set for $M)$.

The proof of Theorem 2.3 given in [22] actually shows the following.

Theorem 2.4. ([22]) For every $M=(\mathbb{N},<, \bar{P})$, there exists $H$ which is uniformly homogeneous for $M$ and recursive in $M S O(M)$.

We shall prove that any set $H$ which is uniformly homogeneous for $M$ and recursive in $M S O(M)$ can be used to expand $M$ and get non-maximality.

Lemma 2.5. For every $M=(\mathbb{N},<, \bar{P})$, if $H$ is uniformly homogeneous for $M$ then $H$ is not definable in $M$.

Proof. Let $H$ be uniformly homogeneous for $M$, and let $h_{0}<h_{1}<\ldots$ be the sequence of elements of $H$. Assume for a contradiction that $H$ is definable in $M$. Then it follows that the set $H_{\text {even }}=\left\{h_{2 i}: i \in \mathbb{N}\right\}$ is also definable in $M$. We shall prove that for every $k \geq 2$, the elements $a_{k}=h_{2 k}$ and $b_{k}=h_{2 k+1}$ satisfy in $M$ the same formulas $\varphi(x)$ with quantifier depth $k$. Since $a_{k} \in H_{\text {even }}$ and $b_{k} \notin H_{\text {even }}$, it follows that $H_{\text {even }}$ is not definable in $M$ by any formula $\varphi(x)$ with quantifier depth less than $k$, from which we get a contradiction.

For every $i \geq 0$ let us denote by $\tau_{i}$ the $i$-type of $M_{\left[h_{i}, h_{i+1}[\right.}$. Let $k \geq 2$. We have $a_{k-1}=h_{2(k-1)}$ and $2(k-1) \geq k$, therefore by definition of $H$ we have

$$
T^{k}\left(M_{\left[a_{k-1}, a_{k}[\right.}\right)=\tau_{k}=T^{k}\left(M_{\left[a_{k-1}, b_{k}[\right.}\right)
$$

thus

$$
\begin{aligned}
T^{k}\left(M_{\left[0, b_{k}[\right.}\right) & =T^{k}\left(M_{\left[0, a_{k-1}[\right.}\right)+T^{k}\left(M_{\left[a_{k-1}, b_{k}[\right.}\right) \\
& =T^{k}\left(M_{\left[0, a_{k-1}[\right.}\right)+T^{k}\left(M_{\left[a_{k-1}, a_{k}[\right.}\right) \\
& =T^{k}\left(M_{\left[0, a_{k}[\right.}\right) .
\end{aligned}
$$

On the other hand by definition of $H$ we also have

$$
T^{k}\left(M_{\left[a_{k}, \infty[\right.}\right)=\tau_{k} \times \omega=T^{k}\left(M_{\left[b_{k}, \infty[\right.}\right) .
$$

It follows from Equations (2.1) and (2.2), and Corollary 1.5 that $a_{k}$ and $b_{k}$ satisfy in $M$ the same formulas $\varphi(x)$ with quantifier depth less than $k$.

Lemma 2.6. Let $H$ be a uniformly homogeneous set for $M=(\mathbb{N},<, \bar{P})$. Then the $M S O$ theory of $M^{\prime}=(\mathbb{N},<, H, \bar{P})$ is recursive in $(H, \bar{P})$.

Proof. Let us denote by $h_{0}<h_{1}<\ldots$ the sequence of elements of $H$. We have

$$
T^{k}\left(M^{\prime}\right)=T^{k}\left(M_{\left[0, h_{k+2}[\right.}^{\prime}\right)+\sum_{i \geq k+2} T^{k}\left(M_{\left[h_{i}, h_{i+1}[\right.}^{\prime}\right)
$$

For every $i \geq k+2$, the only element of $H$ in the interval $\left[h_{i}, h_{i+1}\right.$ [ is $h_{i}$. This implies that for every MSO-sentence $\varphi$ in the signature of $M^{\prime}$ such that $q d(\varphi)=k$, we have

$$
M_{\left[h_{i}, h_{i+1}[\right.}^{\prime} \models \varphi
$$

if and only if

$$
M_{\left[h_{i}, h_{i+1}[\right.} \models \exists x\left((\forall y x \leq y) \wedge \varphi^{*}\right)
$$


where $x$ is any variable which does not appear in $\varphi$, and $\varphi^{*}$ is obtained by replacing in $\varphi$ every atomic formula of the form $H(z)$ (where $z$ denotes any first-order variable) by $z=x$.

The formula in (2.3) has quantifier depth $\leq k+2$. This implies that the $k$-type of $M_{\left[h_{i}, h_{i+1}[\right.}^{\prime}$ can be computed from the $(k+2)$-type of $M_{\left[h_{i}, h_{i+1}[\right.}$. Since $H$ is uniformly homogeneous there is a $(k+2)$-type $\tau_{k+2}$ such that the $(k+2)$-type of $M_{\left[h_{i}, h_{i+1}[\right.}$ equals $\tau_{k+2}$ for every $i \geq k+2$. Moreover, from $H$ and $\bar{P}$ we can compute $h_{k+2}$ and $h_{k+3}$, and then the $(k+2)$-type of $M_{\left[h_{k+2}, h_{k+3}[\right.}$, which equals $\tau_{k+2}$. Thus one can compute $\tau^{\prime}$ such that the $k$-type of $M_{\left[h_{i}, h_{i+1}[\right.}^{\prime}$ equals $\tau^{\prime}$ for every $i \geq k+2$. Therefore we have

$$
T^{k}\left(M^{\prime}\right)=T^{k}\left(M_{\left[0, h_{k+2}[\right.}^{\prime}\right)+\sum_{i \geq k+2} \tau^{\prime}=T^{k}\left(M_{\left[0, h_{k+2}[\right.}^{\prime}\right)+\tau^{\prime} \times \omega
$$

Moreover one checks that $T^{k}\left(M_{\left[0, h_{k+2}[\right.}^{\prime}\right)$ is computable from $H$ and $\bar{P}$.

Finally $T^{k}\left(M^{\prime}\right)$ is computable by Theorem 1.4,

The previous results allow us to show that no structure $M=(\mathbb{N},<, \bar{P})$ is maximal.

Proposition 2.7. For every structure $M=(\mathbb{N},<, \bar{P})$ there exists an expansion $M^{\prime}$ of $M$ by a predicate $P_{n+1}$ such that $P_{n+1}$ is not definable in $M$ and $M S O\left(M^{\prime}\right)$ is recursive in $M S O(M)$. In particular, if $M S O(M)$ is decidable, then $M S O\left(M^{\prime}\right)$ is decidable.

Proof. By Theorem 2.4, there exists a set $H$ which is uniformly homogeneous for $M$ and recursive in $M S O(M)$. We set $P_{n+1}=H$. By Lemma 2.5, $H$ is not definable in $M$. By Lemma 2.6, $M S O\left(M^{\prime}\right)$ is recursive in $H$ and $\bar{P}$, which are both recursive in $M S O(M)$.

In the proof of the general result (see Sect. 4), we start from a labelled linear ordering $M=(A,<, \bar{P})$ and try to define an expansion $M^{\prime}$ such that $M S O\left(M^{\prime}\right)$ is recursive in $M S O(M)$. In some case the expansion $M^{\prime}$ of $M$ will be defined by applying the above construction to infinitely many intervals of $A$ of order type $\omega$. In order to get a reduction from $M S O\left(M^{\prime}\right)$ to $M S O(M)$, we need that the reduction algorithm for such intervals is uniform. This leads to the following Proposition, which can be seen as a uniform version of Proposition 2.7.

Proposition 2.8. There exists a function $E$ and two recursive functions $g_{1}, g_{2}$ such that E maps every structure $M=(\mathbb{N},<, \bar{P})$ to an expansion $M^{\prime}$ of $M$ by a predicate $P_{n+1}$ such that

(1) $P_{n+1}$ is not definable in $M$;

(2) $g_{1}$ computes $T^{k}\left(M^{\prime}\right)$ from $k$ and $T^{g_{2}(k)}(M)$.

Hence $M S O\left(M^{\prime}\right)$ is recursive in $M S O(M)$. In particular, if $M S O(M)$ is decidable, then $M S O\left(M^{\prime}\right)$ is decidable.

The proof of the above proposition relies on the construction of a special uniformly homogeneous set $H$ for $M$.

Proposition 2.9. (22]) There exists a recursive function which maps every $k \geq 0$ to some formula $\varphi_{k}(X)$ such that

(1) for every structure $M=(\mathbb{N},<, \bar{P})$ there exists a unique $X \subseteq \mathbb{N}$ such that $\varphi_{k}(X)$ holds in $M$. This set will be denoted by $H_{k}$.

(2) For every $k \geq 0$, the sets $H_{k}$ and $H_{k+1}$ have the same $k$ first elements.

(3) The set $H=\left\{h_{k}: h_{k}\right.$ is the $k$-th element of $\left.H_{k}\right\}$ is uniformly homogeneous. 
In the above Proposition, for every $i \in \mathbb{N}$ the $i$-th element $h_{i}$ of $H$ is the $i$-th element of the unique set $X \subseteq \mathbb{N}$ such that $\varphi_{i}(X)$ holds in $M$. This implies that for every $i \in \mathbb{N}$, one can compute a formula $\alpha_{i}(x)$ which defines $h_{i}$ in every $M$.

Corollary 2.10. (22]) There exist recursive functions $q$ and $f$ such that for every structure $M=(\mathbb{N},<, \bar{P})$, and corresponding $H$ as in Proposition [2.9, $f$ computes the $i$-th element of $H$ from $i$ and $T^{q(i)}(M)$.

Proof of Proposition 2.8. Let $M=(\mathbb{N},<, \bar{P})$. Let $P_{n+1}=H$ where $H$ is the set associated to $M$ as stated in Proposition 2.9. and let $M^{\prime}$ be the expansion of $M$ by $P_{n+1}$. Let $E$ be the function which maps $M$ to $M^{\prime}$. By Lemma 2.5, $P_{n+1}$ is not MSO definable in $M$.

In order to show that there exist recursive functions $g_{1}, g_{2}$ such that $g_{1}$ computes $T^{k}\left(M^{\prime}\right)$ from $k$ and $T^{g_{2}(k)}(M)$, we have to revise the proof of Lemma 2.6. In this proof the reduction from $T^{k}\left(M^{\prime}\right)$ to $M S O(M)$ comes from the fact that the types $\tau_{k+2}=T^{k+2}\left(M_{\left[h_{k+2}, h_{k+3}[\right.}\right)$, and $T^{k}\left(M_{\left[0, h_{k+2}[\right.}^{\prime}\right)$, are computable from $H$ and $\bar{P}$. We shall prove that for our specific choice of $H$, these types are computable from $T^{g_{2}(k)}(M)$ for some recursive function $g_{2}$.

First, let us consider the type $T^{k+2}\left(M_{\left[h_{k+2}, h_{k+3}[\right.}\right)$. For every sentence $\varphi$ in the signature of $M$ such that $q d(\varphi)=k+2$, we have

$$
M_{\left[h_{k+2}, h_{k+3}[\right.} \models \varphi
$$

if and only if

$$
M \models \exists x \exists y\left(\alpha_{k+2}(x) \wedge \alpha_{k+3}(y) \wedge \varphi^{\prime}\right)
$$

where $x$ and $y$ are variables which do not appear in $\varphi$, and $\varphi^{\prime}$ is obtained from $\varphi$ by relativizing all quantifiers to the interval $[x, y[$. The formula in (2.5) has quantifier depth

$$
q_{1}(k)=2+\max \left(q d\left(\alpha_{k+2}\right), q d\left(\alpha_{k+3}\right), k+2\right)
$$

since $q d\left(\varphi^{\prime}\right)=q d(\varphi)$. The equivalence between (2.4) and (2.5) implies that $\tau_{k+2}$ is computable from $T^{q_{1}(k)}(M)$.

Consider now the type $T^{k}\left(M_{\left[0, h_{k+2}[\right.}^{\prime}\right)$. For every sentence $\varphi$ in the signature of $M^{\prime}$ such that $q d(\varphi)=k$, we have

iff

$$
M_{\left[0, h_{k+2}[\right.}^{\prime} \models \varphi
$$

$$
M \models \exists x\left(\alpha_{k+2}(x) \wedge \varphi^{\prime \prime}\right)
$$

where $x$ is a variable which does not appear in $\varphi$, and $\varphi^{\prime \prime}$ is obtained from $\varphi$ by relativizing all quantifiers to the interval $[0, x[$, and replacing (in the resulting formula) every atomic formula of the form $H(z)$ by the formula $\bigvee_{0 \leq i \leq k+1} \alpha_{i}(z)$. The formula in (2.7) has quantifier depth

$$
q_{2}(k)=1+\max \left(q d\left(\alpha_{k+2}\right), k+\max \left(q d\left(\alpha_{i}\right): 0 \leq i \leq k+1\right)\right) .
$$

The equivalence between (2.6) and (2.7) implies that $T^{k}\left(M_{\left[0, h_{k+2}[\right.}^{\prime}\right)$ is computable from $T^{q_{2}(k)}(M)$.

Finally we obtain that $\tau_{k+2}$ and $T^{k}\left(M_{\left[0, h_{k+2}[\right.}^{\prime}\right)$, and thus $T^{k}\left(M^{\prime}\right)$, are computable from $T^{g_{2}(k)}(M)$ where $g_{2}=\max \left(q_{1}, q_{2}\right)$ is recursive. Moreover the existence of $g_{1}$ clearly follows from the construction. 


\section{The CASe of $\mathbb{Z}$}

Decidability of the MSO theory of structures $M=(\mathbb{Z},<, \bar{P})$ was studied in particular by Compton [9], Semënov [27, 26], and Perrin and Schupp [20] (see also [19, chapter 9]). These works put in evidence the link between decidability of $M S O(M)$ and computability of occurrences and repetitions of finite factors in the word $w(M)$. Let us state some notations and definitions (see e.g. [19, chapter 9]) . A set $X$ of finite words over a finite alphabet $\Sigma$ is said to be regular if it is recognizable by some finite automaton. The length of a finite word $u$ is denoted by $|u|$.

Given a $\mathbb{Z}$-word $w$ and a finite word $u$, both over the alphabet $\Sigma$, we say that $u$ occurs in $w$ if $w=w_{1} u w_{2}$ for some words $w_{1}$ and $w_{2}$. We say that $w$ is recurrent if for every regular language $X$ of finite words over $\Sigma$, either no element of $X$ occurs in $w$, or in every prefix and every suffix of $w$ there is an occurrence of some element of $X$.

In particular in a recurrent word $w$, every finite word $u$ either has no occurrence in $w$, or occurs in every prefix and every suffix of $w$. We say that $w$ is rich if every finite word occurs in every prefix and every suffix of $w$. Given $M=(\mathbb{Z},<, \bar{P})$, we say that $M$ is recurrent if $w(M)$ is.

We have the following result.

Theorem 3.1. (27, 20]) Given $M=\left(\mathbb{Z},<, P_{1}, \ldots, P_{n}\right)$,

(1) If $M$ is not recurrent, then every $c \in \mathbb{Z}$ is definable in $M$.

(2) If $M$ is recurrent, then no element is definable in $M$, and $M S O(M)$ is computable relative to an oracle which, given any regular language $X$ of finite words over $\Sigma_{n}=$ $\{0,1\}^{n}$, tells whether some element of $X$ occurs in $w(M)$.

In this section we prove the following result.

Proposition 3.2. Let $M=\left(\mathbb{Z},<, P_{1}, \ldots, P_{n}\right)$. There exists an expansion $M^{\prime}$ of $M$ by some unary predicate $P_{n+1}$ such that $P_{n+1}$ is not definable in $M$, and $M S O\left(M^{\prime}\right)$ is recursive in $M S O(M)$. In particular, if $M S O(M)$ is decidable, then $M S O\left(M^{\prime}\right)$ is decidable.

Our proof of Proposition 3.2 is based on a definition of $M^{\prime}$ which depends on whether $M$ is recurrent or not. Proposition 3.4 deals with the non-recurrent case, and Proposition 3.6 with the recurrent case. These two propositions yield immediately Proposition 3.2 .

Remark 3.3. Let us discuss uniformity issues related to Proposition 2.8 and Proposition 3.2. Proposition 2.8 implies that there is an algorithm which reduces $M S O\left(M^{\prime}\right)$ to $M S O(M)$. This reduction algorithm is independent of $M$; it only uses an oracle for $M S O(M)$. Proposition 3.2 implies a weaker property. It can be shown indeed that the property for $M$ to be recurrent is not expressible with a MSO sentence in $M$. As a consequence, the algorithm which reduces $M S O\left(M^{\prime}\right)$ to $M S O(M)$ depends on $M$.

3.1. Non-recurrent case. We first deal the case when $M=\left(\mathbb{Z},<, P_{1}, \ldots, P_{n}\right)$ is not recurrent.

Proposition 3.4 (Expansion of non-recurrent structures). There are two recursive functions $g_{1}, g_{2}$ such that if $M=\left(\mathbb{Z},<, P_{1}, \ldots, P_{n}\right)$ is not recurrent, and $c \in \mathbb{Z}$ is definable in $M$ by a formula of quantifier depth $m$, then there exists a function $E_{c}$ which maps $M$ to an expansion $M^{\prime}$ by a predicate $P_{n+1}$ such that

(1) $P_{n+1}$ is not definable in $M$; 
(2) $g_{1}$ computes $T^{k}\left(M^{\prime}\right)$ from $k$ and $T^{g_{2}(k+m)}(M)$.

Hence $M S O\left(M^{\prime}\right)$ is recursive in $M S O(M)$. In particular, if $M S O(M)$ is decidable, then $M S O\left(M^{\prime}\right)$ is decidable.

Proof. Let $c \in \mathbb{Z}$, and let $M_{1}$ be defined as $M_{1}=M_{]-\infty, c[}$ and $M_{2}$ be defined as $M_{[c, \infty[}$. Then $M=M_{1}+M_{2}$. Let $M_{1}^{\prime}$ be the expansion of $M_{1}$ by the empty predicate $P_{n+1}$ and let $M_{2}^{\prime}$ be obtained by applying the construction of Proposition 2.8 to $M_{2}$. Let $M^{\prime}=M_{1}^{\prime}+M_{2}^{\prime}$.

Note that the above construction of $M^{\prime}$ from $M$ depends on $c$. We denote by $E_{c}$ the function described above that maps every $M=\left(\mathbb{Z},<, P_{1}, \ldots, P_{n}\right)$ to its expansion $M^{\prime}$ by $P_{n+1}$.

By definition, $P_{n+1} \cap\left[c, \infty\left[\right.\right.$ is not definable in $M_{2}$, thus $P_{n+1}$ is not definable in $M$ by Proposition 1.6. Hence $M^{\prime}$ is a non-trivial expansion of $M$.

By Theorem 13.1, $c$ is definable in $M$. Hence, $M_{1}$ and $M_{2}$ can be interpreted in $M$, which yields that $\operatorname{MSO}\left(M_{1}\right)$ and $\operatorname{MSO}\left(M_{2}\right)$ are recursive in $\operatorname{MSO}(M)$. Therefore,

$\operatorname{MSO}\left(M_{1}^{\prime}\right)$ and $\operatorname{MSO}\left(M_{2}^{\prime}\right)$ are recursive in $\operatorname{MSO}(M)$. Finally, applying Theorem 1.4(a) we obtain that $\operatorname{MSO}\left(M^{\prime}\right)$ is recursive in $\operatorname{MSO}(M)$.

3.2. Recurrent case. Now we consider the case when $M=(\mathbb{Z},<, \bar{P})$ is recurrent. Let us explain why the construction of the previous sub-section cannot be used in this case. Consider a recurrent structure $M$ and let $M^{\prime}=E_{c}(M)$ for some $c \in \mathbb{Z}$. We claim that it is possible that $M S O\left(M^{\prime}\right)$ is not recursive in $M S O(M)$. Indeed, using ideas from [1] we can prove that there exists a recurrent structure $M$ over $\mathbb{Z}$ such that $M S O(M)$ is decidable, and $M S O\left(M_{\left[c^{\prime}, \infty[\right.}\right)$ is undecidable for every $c^{\prime} \in \mathbb{Z}$. Now let $c^{\prime}$ be the minimal element of $P_{n+1}$. Observe that $c^{\prime}$ is definable in $M^{\prime}$ and therefore, $M_{\left[c^{\prime}, \infty[\right.}$ can be interpreted in $M^{\prime}$. Since, $M S O\left(M_{\left[c^{\prime}, \infty[\right.}\right)$ is undecidable, we derive that $M S O\left(M^{\prime}\right)$ is undecidable. Hence, $E_{c}$ does not preserve decidability of recurrent structures. Thus we need a different construction for the recurrent case.

To describe our construction we introduce first some notations. For every word $w$ over the alphabet $\Sigma_{n+1}=\{0,1\}^{n+1}$ which is indexed by some linear ordering $(A,<)$ we denote by $\pi(w)$ the word $w^{\prime}$ indexed by $A$ and over the alphabet $\Sigma_{n}=\{0,1\}^{n}$, which is obtained from $w$ by projection over the $n$ first components of each symbol in $w$. The definition and notation extend to $\pi(X)$ where $X$ is any set of words over the alphabet $\Sigma_{n+1}$. Given $M=(\mathbb{Z},<, \bar{P})$ where $\bar{P}$ is an $n$-tuple of sets, and any expansion $M^{\prime}$ of $M$ by a predicate $P_{n+1}$, by definition $w(M)$ and $w\left(M^{\prime}\right)$ are words over $\Sigma_{n}$ and $\Sigma_{n+1}$, respectively, and we have $\pi\left(w\left(M^{\prime}\right)\right)=w(M)$.

Lemma 3.5. If $M=\left(\mathbb{Z},<, P_{1}, \ldots, P_{n}\right)$ is recurrent, then there is an expansion $M^{\prime}$ of $M$ by a predicate $P_{n+1}$ which has the following property:

(*): for every $u \in \Sigma_{n}^{*}$, if $u$ occurs in every prefix and every suffix of $w(M)$, then the same holds in $w\left(M^{\prime}\right)$ for every word $u^{\prime} \in \Sigma_{n+1}^{*}$ such that $\pi\left(u^{\prime}\right)=u$.

Proof. The proof is similar to the proof of Proposition 2.8 in [1], which roughly shows how to deal with the case when $w(M)$ is rich.

Let $X$ be the set of nonempty words $u \in \Sigma_{n+1}^{*}$ such that $\pi(u)$ occurs in (every prefix and every suffix of ) $w(M)$. We define the expansion $M^{\prime}$ of $M$ by $P_{n+1}$ in such a way that every element of $X$ occurs in every prefix and every suffix of $w\left(M^{\prime}\right)$. This can be done as follows. 
We first define the restriction of $P_{n+1}$ to the interval [0, $\infty\left[\right.$. Let $\left(u_{i}\right)_{i \in \mathbb{N}}$ be any sequence of elements of $\Sigma_{n+1}^{*}$ such that $X=\left\{u_{i}: i \geq 0\right\}$, and for every $u \in X$ the set $\left\{i: u_{i}=u\right\}$ is infinite. We shall define sequences of integers $\left(a_{m}\right)_{m \in \mathbb{N}}$ and $\left(b_{m}\right)_{m \in \mathbb{N}}$ and the restriction of $P_{n+1}$ to $\left[0, b_{m}\right]$ in such a way that each $u_{m}$ occurs in $w\left(M_{\left[0, b_{m}\right]}^{\prime}\right)$, and $a_{m}$ corresponds to some position in $\left[0, b_{m}\right]$ at which $u_{m}$ starts. We proceed by induction over $m$.

- Case $m=0$ : we have $u_{0} \in X$, therefore the word $\pi\left(u_{0}\right)$ has an occurrence in $w\left(M_{[0, \infty}\right)$. Let $a_{0} \geq 0$ be the least non-negative integer such that $\pi\left(u_{0}\right)=w\left(M_{\left[a_{0}, a_{0}+\left|u_{0}\right|[\right.}\right)$, and let $b_{0}=a_{0}+\left|u_{0}\right|-1$. We define the restriction of $P_{n+1}$ to $\left[a_{0}, a_{0}+\left|u_{0}\right|[\right.$ such that $w\left(M_{\left[a_{0}, a_{0}+\left|u_{0}\right|[\right.}^{\prime}\right)=u_{0}$. Moreover we set $P_{n+1} \cap\left[0, a_{0}[=\varnothing\right.$.

- Induction step: let $m \geq 1$. We have $u_{m} \in X$, therefore the word $\pi\left(u_{m}\right)$ has an occurrence in $w\left(M_{\left[b_{m-1}, \infty[\right.}\right)$. We define $a_{m}$ as the least integer greater than $b_{m-1}$ such that $\pi\left(u_{m}\right)=w\left(M_{\left[a_{m}, a_{m}+\left|u_{m}\right|[\right.}\right)$. We set $b_{m}=a_{m}+\left|u_{m}\right|-1$. We then define the restriction of $P_{n+1}$ to $\left[a_{m}, a_{m}+\left|u_{m}\right|\left[\right.\right.$ in such a way that $w\left(M_{\left[a_{m}, a_{m}+\left|u_{m}\right|[\right.}^{\prime}\right)=u_{i}$. Moreover we set $\left.P_{n+1} \cap\right] b_{m-1}, a_{m}[=\varnothing$.

This construction, and the definition of the sequence $\left(u_{i}\right)$, guarantee that every word $u \in X$ occurs in every suffix of $w\left(M^{\prime}\right)$.

We can proceed in a similar way for the definition of the restriction of $P_{n+1}$ to the interval $]-\infty, 0\left[\right.$, in such a way that every word $u \in X$ occurs in every prefix of $w\left(M^{\prime}\right)$.

Proposition 3.6 (Expansion of recurrent structures). There are two recursive function $g_{1}, g_{2}$ such that if $M=\left(\mathbb{Z},<, P_{1}, \ldots, P_{n}\right)$ is recurrent and $M^{\prime}$ is an expansion of $M$ which has property $\left({ }^{*}\right)$, then

(1) $P_{n+1}$ is not definable in $M$;

(2) $g_{1}$ computes $T^{k}\left(M^{\prime}\right)$ from $k$ and $T^{g_{2}(k)}(M)$.

Hence $M S O\left(M^{\prime}\right)$ is recursive in $M S O(M)$. In particular, if $M S O(M)$ is decidable, then $M S O\left(M^{\prime}\right)$ is decidable.

Remark 3.7. Proposition 3.6 implies that there is an algorithm which reduces $M S O\left(M^{\prime}\right)$ to $M S O(M)$. This reduction algorithm (like the algorithm from Proposition 2.8) is independent of $M$; it only uses an oracle for $M S O(M)$.

The proof of Proposition 3.6 relies on a refinement of Theorem 3.1. The analysis of Semënov's proof [27] of the latter shows that for a given $k \geq 0$, the $k$-type of $M S O(M)$ can actually be computed as soon as we can decide, for finitely many regular languages $L$ (which can be computed from $k$ ), whether some element of $L$ occurs in $w(M)$. More precisely we have the following.

Proposition 3.8. (Semënov [27], Perrin-Schupp [20]) There exists a recursive function which maps every $k \geq 0$ to a finite sequence $S_{k}=\left(L_{k, 0}, \ldots, L_{k, a_{k}}\right)$ of regular languages of finite words over $\Sigma_{n}$ such that for every structure $M=\left(\mathbb{Z},<, P_{1}, \ldots, P_{n}\right)$ with w(M) recurrent, $T^{k}(M)$ is computable relative to the set

$$
I_{k}=\left\{i \text { : some element of } L_{k, i} \text { occurs in } w(M)\right\} .
$$

\section{Proof of Proposition 3.6.}

Let $M=\left(\mathbb{Z},<, P_{1}, \ldots, P_{n}\right)$ be recurrent, and let $M^{\prime}$ be an expansion of $M$ which has property $(*)$.

First let us prove that $P_{n+1}$ is not definable in $M$. For simplicity we use the notations $w, w^{\prime}$ for $w(M), w\left(M^{\prime}\right)$, respectively. For a fixed $k \geq 0$ there exist finitely many $(k,\{<$ 
$\left.\left., P_{1}, \ldots, P_{n}\right\}\right)$-Hintikka sentences, say $m_{k}$. Let $u$ be any finite word over $\Sigma_{n}$ such that $u$ is recurrent in $w$ and $2^{|u|}>\left(m_{k}\right)^{2}$. Since $u$ is recurrent in $w$, it follows from the construction of $w^{\prime}$ that every word $u^{\prime}$ over $\Sigma_{n+1}$ such that $\pi\left(u^{\prime}\right)=u$ is also recurrent in $w^{\prime}$. There are $2^{|u|}$ distinct such words, say $u_{1}^{\prime}, \ldots, u_{2^{|u|}}^{\prime}$. For $i=1, \ldots, 2^{|u|}$, let $\left(s_{i}, t_{i}\right)$ be such that $w^{\prime}\left(\left[s_{i}, t_{i}[)=\right.\right.$ $u_{i}^{\prime}$ (which implies $w\left(\left[s_{i}, t_{i}[)=u\right.\right.$ ). By definition of $u$ we have $2^{|u|}>\left(m_{k}\right)^{2}$, which implies that there exist $i<j$ such that $T^{k}\left(M_{\left[0, s_{i}[\right.}\right)=T^{k}\left(M_{\left[0, s_{j}[\right.}\right)$ and $T^{k}\left(M_{\left[t_{i}, \infty[\right.}\right)=T^{k}\left(M_{\left[t_{j}, \infty[\right.}\right)$. We have $w\left(\left[s_{i}, t_{i}[)=w\left(\left[s_{j}, t_{j}[)=u\right.\right.\right.\right.$, hence the $T^{k}\left(M_{\left[s_{i}, t_{i}\right.}[)=T^{k}\left(M_{\left[s_{j}, t_{j}\right.}\right]\right)$. It follows from $w^{\prime}\left(\left[s_{i}, t_{i}[) \neq w^{\prime}\left(\left[s_{j}, t_{j}[)\right.\right.\right.\right.$ that there exists $l \in\left\{0, t_{i}-s_{i}-1\right\}$ such that $w^{\prime}\left(s_{i}+l\right) \neq w^{\prime}\left(s_{j}+l\right)$ (where $w^{\prime}(x)$ denotes the letter at position $x$ in $w^{\prime}$ ). Assume that $l \notin\left\{0, t_{i}-s_{i}-1\right\}$ (the case $\left.l \in\left\{0, t_{i}-s_{i}-1\right\}\right)$ is similar). that

We have $w\left(\left[s_{i}, s_{i}+l[)=w\left(\left[s_{j}, s_{j}+l[)\right.\right.\right.\right.$ thus $T^{k}\left(M_{\left[s_{i}, s_{i}+l[\right.}\right)=T^{k}\left(M_{\left[s_{j}, s_{j}+l[\right.}\right)$. It follows

$$
\begin{aligned}
T^{k}\left(M_{\left[0, s_{i}+l[\right.}\right) & =T^{k}\left(M_{\left[0, s_{i}[\right.}\right)+T^{k}\left(M_{\left[s_{i}, s_{i}+l[\right.}\right) \\
& =T^{k}\left(M_{\left[0, s_{j}[\right.}\right)+T^{k}\left(M_{\left[s_{j}, s_{j}+l[\right.}\right) \\
& =T^{k}\left(M_{\left[0, s_{j}+l[\right.}\right) .
\end{aligned}
$$

Moreover $w\left(\left[s_{i}+l, t_{i}[)=w\left(\left[s_{j}+l, t_{j}[)\right.\right.\right.\right.$ thus $T^{k}\left(M_{\left[s_{i}+l, t_{i}[\right.}\right)=T^{k}\left(M_{\left[s_{j}+l, t_{j}[\right.}\right)$. Hence

$$
\begin{aligned}
T^{k}\left(M_{\left[s_{i}+l, \infty[\right.}\right) & =T^{k}\left(M_{\left[s_{i}+l, t_{i}[\right.}\right)+T^{k}\left(M_{\left[t_{i}, \infty[\right.}\right) \\
& =T^{k}\left(M_{\left[s_{j}+l, t_{j}\right.}\right)+T^{k}\left(M_{\left[t_{j}, \infty[\right.}\right) \\
& =T^{k}\left(M_{\left[s_{j}+l, \infty[\right.}\right)
\end{aligned}
$$

If $k \geq 1$, it follows from (3.1), (3.2) and Corollary 1.5 that the elements $s_{i}+l$ and $s_{j}+l$ cannot be distinguished by any formula $\varphi(x)$ with quantifier depth less than $k$. Since $w^{\prime}\left(s_{i}+l\right) \neq w^{\prime}\left(s_{j}+l\right)$ this implies that $P_{n+1}$ is not definable by any formula of quantifier depth less than $k$. Since this holds for every $k$, we conclude that $P_{n+1}$ is not definable in $M$.

Let us now prove that one can compute $T^{k}\left(M^{\prime}\right)$ from $k$ and $T^{g_{2}(k)}(M)$, for some recursive function $g_{2}$. Let $k \geq 1$. Using Proposition 3.8 we can compute $S_{k}=\left(L_{k, 0}^{\prime}, \ldots, L_{k, a_{k}}^{\prime}\right)$ such that $T^{k}\left(M^{\prime}\right)$ is computable relative to the set

$$
I_{k}=\left\{i: \text { some element of } L_{k, i}^{\prime} \text { occurs in } w\left(M^{\prime}\right)\right\} \text {. }
$$

Observe that $P_{n+1}$ satisfies (*), thus for every regular language $X^{\prime} \subseteq \Sigma_{n+1}^{*}$, some element of $X$ occurs in $w\left(M^{\prime}\right)$ iff some element of $\pi(X)$ occurs in $w\left(M^{\prime}\right)$. It follows that if we set $L_{k, i}=\pi\left(L_{k, i}^{\prime}\right)$ for every $i$, then we have

$$
I_{k}=\left\{i: \text { some element of } L_{k, i} \text { occurs in } w(M)\right\} .
$$

Every language $L_{k, i}^{\prime}$ is regular, and thus the same holds for $L_{k, i}$. Given any $L_{k, i}$, we can compute a sentence $F_{i}$ such that $M \models F_{i}$ iff some element of $L_{k, i}$ occurs in $w(M)$. It follows that $I_{k}$ is recursive in $M S O(M)$. Moreover the computation involves only deciding sentences of the form $F_{i}$, i.e. uses an oracle for $T^{g_{2}(k)}(M)$ where $g_{2}(k)$ is the maximal quantifier depth of sentences of the form $F_{i}$. This completes the proof. 


\section{Main Result}

The next theorem is our main result.

Theorem 4.1. Let $M=\left(A,<, P_{1}, \ldots, P_{n}\right)$ where $(A,<)$ contains an interval of type $\omega$ or $-\omega$. There exists an expansion $M^{\prime}$ of $M$ by a unary predicate $P_{n+1}$ such that $P_{n+1}$ is not definable in $M$, and $M S O\left(M^{\prime}\right)$ is recursive in $M S O(M)$. In particular, if $M S O(M)$ is decidable, then $M S O\left(M^{\prime}\right)$ is decidable.

As an immediate consequence we obtain the following corollary.

Corollary 4.2. Let $M=\left(A,<, P_{1}, \ldots, P_{n}\right)$ where $(A,<)$ is an infinite scattered linear ordering. There exists an expansion $M^{\prime}$ of $M$ by some unary predicate $P_{n+1}$ not definable in $M$ such that $M S O\left(M^{\prime}\right)$ is recursive in $M S O(M)$.

We shall use Shelah's composition method [28, Theorem 2.4] (see also [14, 32]) which allows us to reduce the MSO theory of a sum of chains to the MSO theories of the summands and the MSO theory of the index structure.

Theorem 4.3 (Composition Theorem [28]). There exists a recursive function $f$ and an algorithm which, given $k, l \in \mathbb{N}$, computes the $k$-type of any sum $M=\sum_{i \in I} M_{i}$ of chains over a signature $\left\{<, P_{1}, \ldots, P_{l}\right\}$ from the $f(k, l)$-type of the structure

$$
\left(I,<^{I}, Q_{1}, \ldots, Q_{p}\right)
$$

where

$$
Q_{j}=\left\{i \in I: T^{k}\left(M_{i}\right)=\tau_{j}\right\} \quad j=1, \ldots, p
$$

and $\tau_{1}, \ldots, \tau_{p}$ is the list of all $(k, \Delta)$-Hintikka sentences with $\Delta=\left\{<, P_{1}, \ldots, P_{l}\right\}$.

\section{Proof of Theorem 4.1.}

Let $M=(A,<, \bar{P})$ where $(A,<)$ contains an interval of type $\omega$ or $-\omega$. The main idea is to use a definable equivalence relation on $A$ to cut $A$ into intervals which are either finite, or have order type $\omega,-\omega$ or $\zeta$, and then use the previous constructions to define $P_{n+1}$ in these intervals.

Consider the binary relation defined on $A$ by $x \approx y$ iff $[x, y]$ is finite. The relation $\approx$ is a convex equivalence relation, and is definable in $M$.

Let $I$ be the linear order of the $\approx$-equivalence classes for $(A,<)$. Then $M=\sum_{i \in I} M_{A_{i}}$ where the $A_{i}$ 's correspond to equivalence classes of $\approx$. Using the definition of $\approx$ and our assumption on $A$, it is easy to check that the $A_{i}$ 's are either finite, or of order type $\omega$, or $-\omega$, or $\zeta$, and that not all $A_{i}$ 's are finite.

We define the interpretation of the new predicate $P_{n+1}$ in every interval $A_{i}$. The definition proceeds as follows:

(1) if some $A_{i}$ has order type $\omega$ or $-\omega$, then we consider two subcases:

(a) if some $A_{i}$ has order type $\omega$, then we apply to each substructure $M_{A_{i}}$ of order type $\omega$ the construction given in Proposition 2.8, and add no element of $P_{n+1}$ elsewhere.

(b) if no $A_{i}$ has order type $\omega$, we proceed in a similar way with each substructure $M_{A_{i}}$ of order type $-\omega$, but using the dual of Proposition 2.8 for $-\omega$.

(2) if no $A_{i}$ has order type $\omega$ or $-\omega$, then at least one $\approx$-equivalence class $A_{i}$ has order type $\zeta$. We consider two subcases:

(a) if all $A_{i}$ with order type $\zeta$ are such that $M_{A_{i}}$ is recurrent, then we apply to each substructure $M_{A_{i}}$ of order type $\zeta$ the construction given in Proposition 3.6, and add no element of $P_{n+1}$ elsewhere. 
(b) otherwise there exists some $A_{i}$ with order type $\zeta$ and such that $M_{A_{i}}$ is not recurrent. Let $\varphi(x)$ be a formula with minimal quantifier depth such that $\varphi(x)$ defines an element in some $M_{A_{i}}$ where $A_{i}$ has order type $\zeta$. For every $M_{A_{i}}$ such that $A_{i}$ has order type $\zeta$ and $\varphi(x)$ defines an element $c_{i}$ in $M_{A_{i}}$, we apply the construction $E_{c_{i}}$ from Proposition 3.4 to $M_{A_{i}}$, and add no element of $P_{n+1}$ elsewhere.

Lemma 4.4. $P_{n+1}$ is not definable in $M$.

Proof. This is easy to deduce from the construction: by Proposition 1.6, if $P_{n+1}$ was definable in $M$ then the same would hold for $P_{n+1} \cap A_{i}$ in every sub-structure $M_{A_{i}}$.

If we are in case (1), that is, if some $A_{i}$ has order type $\omega$ or $-\omega$, then we applied the construction of Proposition 2.8 to at least one substructure $M_{A_{j}}$, therefore $P_{n+1} \cap A_{j}$ is not definable in $M_{A_{j}}$, which leads to a contradiction. Case $2(b)$ is similar. For case $2(a)$ the contradiction arises from Proposition 3.4 instead of Proposition 2.8.

Lemma 4.5. Let $M^{\prime}$ be the expansion of $M$ by the predicate $P_{n+1}$. Then $M S O\left(M^{\prime}\right)$ is recursive in $M S O(M)$.

Proof. For every $i \in I$ we denote by $M_{i}^{\prime}$ (respectively $M_{i}$ ) the substructure of $M^{\prime}$ (resp. $M$ ) with domain $A_{i}$. Let $k \geq 1$, and let us compute $T^{k}\left(M^{\prime}\right)$. By Theorem 4.3, $T^{k}\left(M^{\prime}\right)$ can be computed from the $f(k, n+1)$-type of the structure $\left(I,<, Q_{1}^{\prime}, \ldots, Q_{p}^{\prime}\right)$ where

$$
Q_{j}^{\prime}=\left\{i \in I: T^{k}\left(M_{i}^{\prime}\right)=\tau_{j}^{\prime}\right\} \quad j=1, \ldots, p
$$

and $\tau_{1}^{\prime}, \ldots, \tau_{p}^{\prime}$ denote the list of all $\left(k, \Delta^{\prime}\right)$-Hintikka sentences with $\Delta^{\prime}=\left\{<, \bar{P}, P_{n+1}\right\}$.

Let $l=f(k, n+1)$. Let us prove that for every $i \in I$, the $l$-type of $M_{i}^{\prime}$ can be computed from the $g(l)$-type of $M_{i}$ for some recursive function $g$. Note that $g$ depends on $M$, namely whether we used case (1) or (2) to construct $M^{\prime}$.

Assume first that we are in case $(1)(a)$. In this case we first check whether the order type of the domain $A_{i}$ of $M_{i}$ has order type $\omega$; that is, we check whether $M_{i} \models F$ where $F$ expresses that the domain of the structure has order type $\omega$. If the answer is positive, then it means that $M_{i}^{\prime}$ was defined by using the construction given in the proof of Proposition 2.8, and thus it follows from item (2) in the latter proposition that $T^{l}\left(M_{i}^{\prime}\right)$ is computable from $T^{g^{2}(l)}\left(M_{i}\right)$. Otherwise we have set $P_{n+1} \cap A_{i}=\varnothing$, and in this case the $l$-type of $M_{i}^{\prime}$ can be computed directly from the $l$-type of $M_{i}$.

Case $(1)(b)$ is similar to the previous one.

For Case $(2)(a)$, we first check whether the order type of the domain $A_{i}$ is $\zeta$. If the answer is positive, then it means that $M_{i}^{\prime}$ was defined by using the construction given in the proof of Proposition 3.6, and thus the reasoning is similar to the one for case $(1)(a)$.

For Case $(2)(b)$, we first check whether the order type of the domain $A_{i}$ is $\zeta$. If the answer is positive, then we also check whether the formula $\varphi(x)$ (which was used in the description of case 2(b)) defines an element in $M_{i}$. Namely, we test whether the sentence $\psi \equiv \exists x(\varphi(x) \wedge \forall y(\varphi(y) \rightarrow y=x))$ holds in $M_{i}$. Note that $q d(\psi)=l+2$. If the answer is positive again, then it means that $M_{i}^{\prime}$ was defined by using the construction given in the proof of Proposition 3.4, which implies that the $l$-type of $M_{i}^{\prime}$ is computable from the $r$-type of $M_{i}$, with $r=\max \left(l+2, g_{2}(l+k)\right)$. Otherwise we have set $P_{n+1} \cap A_{i}=\varnothing$, and in this case the $l$-type of $M_{i}^{\prime}$ can be computed directly from the $l$-type of $M_{i}$.

Since for every $i \in I, T^{l}\left(M_{i}^{\prime}\right)$ is computable from $T^{g(l)}\left(M_{j}\right)$ for some recursive function $g$, every formula $Q_{i}^{\prime}(x)$ is equivalent to a boolean combination of predicates of the form 
$Q_{j}(x)$ where

$$
Q_{j}=\left\{i \in I: T^{g(l)}\left(M_{i}\right)=\tau_{j}\right\} \quad j=1, \ldots, r
$$

and $\tau_{1}, \ldots, \tau_{r}$ denote the sequence of $(g(l), \Delta)$-Hintikka sentences with $\Delta=\{<, \bar{P}\}$.

It follows that $\left(I,<, Q_{1}^{\prime}, \ldots, Q_{p}^{\prime}\right)$ is interpretable in the structure $N=\left(I,<, Q_{1}, \ldots, Q_{r}\right)$, and Lemma 1.1 yields that $M S O\left(I,<, Q_{1}^{\prime}, \ldots, Q_{p}^{\prime}\right)$ is recursive in $M S O(N)$. Now the relation $\approx$ is a convex equivalence relation and is definable in $M$. Thus by Lemma 1.7, the structure $N$ is interpretable in $M$, and $M S O(N)$ is recursive in $M S O(M)$ by Lemma 1.1. $\square$

Theorem 4.1 follows from Lemmas 4.4 and 4.5 .

Remark 4.6. Let us discuss uniformity issues related to Theorem 4.1 .

- The choice to expand "uniformly" all $\approx$-equivalence classes is crucial for the reduction from $M S O\left(M^{\prime}\right)$ to $M S O(M)$. For example, if some $A_{i}$ has order type $\omega$ and we choose to expand only one such $A_{i}$ then $M S O\left(M^{\prime}\right)$ might become undecidable. This is the case for the structure $M$ considered in [2] (Definition 2.4), which has decidable MSO theory, and is such that the MSO (and even FO) theory of any expansion of $M$ by a constant is undecidable. For this structure all $A_{i}$ 's have order type $\omega$. If we consider the structure $M^{\prime}$ obtained from $M$ by an expansion of only one $A_{i}$, then $P_{n+1}$ has a least element, which is definable in $M^{\prime}$, thus $\operatorname{MSO}\left(M^{\prime}\right)$ is undecidable.

- The definition of $P_{n+1}$ in case (2) depends on whether all components $A_{i}$ with order type $\zeta$ are such that $w\left(M_{A_{i}}\right)$ is recurrent, which is not a MSO definable property. Thus the reduction algorithm from $M S O\left(M^{\prime}\right)$ to $M S O(M)$ depends on $M$.

\section{Further Results and Open Questions}

Let us mention some possible extensions and related open questions.

First of all, most of our results can be easily extended to the case when the signature contains infinitely many unary predicates.

Our results can be extended to the Weak MSO logic. In the case $M$ is countable this follows from Soprunov result [30]. However, our construction works for labelled orderings of arbitrary cardinality.

An interesting issue is to prove uniform versions of our results in the sense of items (2) in Propositions 2.8 and 3.6. A first step would be to generalize Proposition 3.6 to all structures $(\mathbb{Z},<, \bar{P})$.

One can also ask whether the results of the present paper hold for FO logic. Let us emphasize some difficulties which arise when one tries to adapt the main arguments. A FO version of Theorem 2.3 (about the recursive homogeneous set) was already proven in 23]. Moreover, using ideas from [27] one can also give a characterization of structures $M=(\mathbb{Z},<, \bar{P})$ with a decidable FO theory, in terms of occurrences and repetitions of finite words in $w(M)$. This allows us to give a $\mathrm{FO}$ version of our non-maximality results for labelled orders over $\omega$ or $\zeta$. However for the general case of $(A,<, \bar{P})$, two problems arise: (1) the constructions for $\mathbb{N}$ and $\mathbb{Z}$ cannot be applied directly since they are not uniform, and $(2)$ the equivalence relation $\approx$ used in the proof of Theorem 4.1 to cut $A$ into small intervals is not FO definable. We currently investigate these issues.

Finally, we also study the case of labelled linear orderings $(A,<, \bar{P})$ which do not contain intervals of order types $\omega$ or $-\omega$. In this case the construction presented in Sect. 4 does not work since the restriction of $P_{n+1}$ to each $A_{i}$ will be empty, i.e., our new relation is 
actually empty. In a forthcoming paper we show that it is possible to overcome this issue for countable orders, and prove that no infinite countable structure $(A,<, \bar{P})$ is maximal.

Acknowledgment. We thank the anonymous referees for useful suggestions.

\section{REFERENCES}

[1] A. Bès and P. Cégielski. Weakly maximal decidable structures. RAIRO-Theor. Inf. Appl., 42(1):137-145, 2008.

[2] A. Bès and P. Cégielski. Nonmaximal decidable structures. Journal of Mathematical Sciences, 158:615$622,2009$.

[3] A. Bès and A. Rabinovich. Decidable expansions of labelled linear orderings. In YuriFest, Symposium on Logic in Computer Science Celebrating Yuri Gurevich Seventieth Birthday, LNCS, 2010. to appear.

[4] A. Blumensath, T. Colcombet, and C. Löding. Logical theories and compatible operations. In J. Flum, E. Grädel, and T. Wilke, editors, Logic and automata: History and Perspectives, pages 72-106. Amsterdam University Press, 2007.

[5] J. R. Büchi. On a decision method in the restricted second-order arithmetic. In Proc. Int. Congress Logic, Methodology and Philosophy of science, Berkeley 1960, pages 1-11. Stanford University Press, 1962.

[6] J. R. Büchi. Transfinite automata recursions and weak second order theory of ordinals. In Proc. Int. Congress Logic, Methodology, and Philosophy of Science, Jerusalem 1964, pages 2-23. North Holland, 1965.

[7] J. R. Büchi and C. Zaiontz. Deterministic automata and the monadic theory of ordinals $\omega_{2}$. Z. Math. Logik Grundlagen Math., 29:313-336, 1983.

[8] O. Carton and W. Thomas. The monadic theory of morphic infinite words and generalizations. Inform. Comput., 176:51-76, 2002.

[9] K. J. Compton. On rich words. In M. Lothaire, editor, Combinatorics on words. Progress and perspectives, Proc. Int. Meet., Waterloo/Can. 1982, volume 17 of Encyclopedia of Mathematics, pages 39-61. Addison-Wesley, 1983.

[10] C. C. Elgot and M. O. Rabin. Decidability and undecidability of extensions of second (first) order theory of (generalized) successor. J. Symb. Log., 31(2):169-181, 1966.

[11] S. Fratani. The theory of successor extended with several predicates. preprint, 2009.

[12] D. Gabbay. The declarative past and imperative future. In B. Banieqbal, H. Barringer, and A. Pnueli, editors, Temporal Logic in Specification, volume 398 of Lecture Notes in Computer Science, pages 409448. Springer Berlin / Heidelberg, 1989.

[13] Y. Gurevich. Modest theory of short chains. i. J. Symb. Log., 44(4):481-490, 1979.

[14] Y. Gurevich. Monadic second-order theories. In J. Barwise and S. Feferman, editors, Model-Theoretic Logics, pages 479-506. Springer-Verlag, Perspectives in Mathematical Logic, 1985.

[15] Y. Gurevich, M. Magidor, and S. Shelah. The monadic theory of $\omega_{2}$. J. Symb. Log, 48(2):387-398, 1983.

[16] Y. Gurevich and S. Shelah. Modest theory of short chains. ii. J. Symb. Log., 44(4):491-502, 1979.

[17] Y. Gurevich and S. Shelah. Interpreting second-order logic in the monadic theory of order. J. Symb. Log., 48(3):816-828, 1983.

[18] J. A. Makowsky. Algorithmic uses of the Feferman-Vaught theorem. Annals of Pure and Applied Logic, 126(1-3):159-213, 2004.

[19] D. Perrin and J.-E. Pin. Infinite Words, volume 141 of Pure and Applied Mathematics. Elsevier, 2004.

[20] D. Perrin and P. E. Schupp. Automata on the integers, recurrence distinguishability, and the equivalence and decidability of monadic theories. In Symposium on Logic in Computer Science (LICS '86), pages 301-305, Washington, D.C., USA, June 1986. IEEE Computer Society Press.

[21] M.O. Rabin. Decidability of second-order theories and automata on infinite trees. Transactions of the American Mathematical Society, 141:1-35, 1969.

[22] A. Rabinovich. On decidability of monadic logic of order over the naturals extended by monadic predicates. Inf. Comput, 205(6):870-889, 2007. 
[23] A. Rabinovich and W. Thomas. Decidable theories of the ordering of natural numbers with unary predicates. In Zoltán Ésik, editor, Computer Science Logic, 20th International Workshop, CSL 2006, 15th Annual Conference of the EACSL, Szeged, Hungary, September 25-29, 2006, Proceedings, volume 4207 of Lecture Notes in Computer Science, pages 562-574. Springer, 2006.

[24] R.M. Robinson. Restricted set-theoretical definitions in arithmetic. Proc. Am. Math. Soc., 9:238-242, 1958.

[25] J. G. Rosenstein. Linear ordering. Academic Press, New York, 1982.

[26] A. L. Semenov. Decidability of monadic theories. In M. P. Chytil and V. Koubek, editors, Proceedings of the 11th Symposium on Mathematical Foundations of Computer Science, volume 176 of LNCS, pages 162-175, Praha, Czechoslovakia, September 1984. Springer.

[27] A. L. Semenov. Logical theories of one-place functions on the set of natural numbers. Mathematics of the USSR - Izvestia, 22:587-618, 1984.

[28] S. Shelah. The monadic theory of order. Annals of Mathematics, 102:379-419, 1975.

[29] D. Siefkes. Decidable extensions of monadic second order successor arithmetic. Automatentheorie und Formale Sprachen, (Tagung, Math. Forschungsinst, Oberwolfach), 1969; (Bibliograph. Inst., Mannheim), pages 441-472, 1970.

[30] S. Soprunov. Decidable expansions of structures. Vopr. Kibern., 134:175-179, 1988. (in Russian).

[31] W. Thomas. A note on undecidable extensions of monadic second order successor arithmetic. Arch. Math. Logik Grundlagenforsch., 17:43-44, 1975.

[32] W. Thomas. Ehrenfeucht games, the composition method, and the monadic theory of ordinal words. In Structures in Logic and Computer Science, A Selection of Essays in Honor of A. Ehrenfeucht, number 1261 in LNCS, pages 118-143. Springer, 1997.

[33] W. Thomas. Languages, automata, and logic. In G. Rozenberg and A. Salomaa, editors, Handbook of Formal Languages, volume III, pages 389-455. Springer-Verlag, 1997.

[34] W. Thomas. Model transformations in decidability proofs for monadic theories. In Michael Kaminski and Simone Martini, editors, CSL, volume 5213 of Lecture Notes in Computer Science, pages 23-31. Springer, 2008. 